Chirurg 2020 $91: 354$

https://doi.org/10.1007/s00104-020-01128-4

Online publiziert: 4. Februar 2020

(c) Springer Medizin Verlag $\mathrm{GmbH}$, ein Teil von Springer Nature 2020

\author{
Henning Dralle ${ }^{1}$ Theresia Weber ${ }^{2}$ \\ 'Sektion Endokrine Chirurgie, Klinik für Allgemein-, Viszeral- und Transplantationschirurgie, \\ Universitätsklinikum Essen, Essen, Deutschland \\ ${ }^{2}$ Klinik für Endokrine Chirurgie, Katholisches Klinikum Mainz, Mainz, Deutschland
}

\title{
Parathyreoidektomie in der Schwangerschaft
}

ser oder operativer Therapie des pHPT wurden analysiert. Da der Zeitpunkt der Diagnose des pHPT die Therapieentscheidung für ein operatives Vorgehen beeinflusst haben konnte, wurden 3 Gruppen gebildet: medikamentöse Therapie vor oder nach der 28. Schwangerschaftswoche (K28) und chirurgische Therapie im 2. Trimenon.

Hintergrund. Ein primärer Hyperparathyreoidismus (pHPT) in der Schwangerschaft wird bei ca. 1,4\% der Frauen beobachtet. Da sowohl maternale (Präeklampsie; Hyperemesis gravidarum; hyperkalzämische Krise) als auch fetale Komplikationen (neonatale Hypokalzämie mit Tetanie; Früh-, Fehl- oder Totgeburt; intrauterine Wachstumsverzögerung; erniedrigtes Geburtsgewicht) berichtet werden, ist im Einzelfall die Entscheidung hinsichtlich einer medikamentösen oder operativen Therapie schwierig. Allgemein ist davon auszugehen, dass mit Anstieg der maternalen Serumkalziumkonzentrationen das krankheitsbedingte Komplikationsrisiko zunimmt, ein Konsens über Grenzwerte, bei deren Erreichen bzw. Überschreiten ein operatives Vorgehen zu favorisieren ist, besteht jedoch bislang nicht. Die vorliegende retrospektive Studie berichtet über 28 Patientinnen mit pHPT in der Schwangerschaft, 22 wurden medikamentös, 6 operativ behandelt.

Material und Methoden. Die Analyse der Krankengeschichten des australischen Krankenhauses in Brisbane ergab 28 Frauen mit laborchemisch gesichertem pHPT. Alle verfügbaren Daten zur Laborchemie, dem Verlauf der Schwangerschaft und Geburt und dem Outcome der Neugeborenen nach medikamentö-
Ergebnisse. Das mittlere Alter der Frauen betrug 30 bis 36 Jahre. Die mittleren initialen bzw. höchsten Serumkalziumwerte lagen in den 3 Gruppen bei 2,7-2,9 bzw. 2,8-3,1 mmol/l. Die 6 parathyreoidektomierten Patientinnen hatten ein Adenom mit postoperativer Normalisierung des Kalziums und Parathormons. Bei den medikamentös behandelten Patientinnen traten folgende Komplikationen auf: Präeklampsie (6/22; 30\%); Schwangerschaftshypertension $\quad(3 / 22$; $15 \%)$; vorzeitige Geburtseinleitung aufgrund Präeklampsie (4/22; $20 \%)$; Fehlgeburt $(2 / 22 ; 10 \%)$; bei den chirurgisch behandelten Patientinnen: keine Präeklampsie, 2 Fälle von Schwangerschaftshypertension, kein Fall vorzeitiger Geburt. Neugeborene: 4/22 Babys der medikamentös behandelte Patientinnen erforderten eine Intensivbehandlung wegen Frühreife nach vorzeitiger Geburt, eins dieser Babys hatte eine milde Hypokalzämie. Nach Parathyreoidektomie musste ein zum Termin geborenes Kind wegen pulmonaler Hypertension intensivbehandelt werden. Ansonsten traten keine operationsbezogenen Komplikationen auf.

\section{Kommentar}

Vorteil der Studie ist die sorgfältige Bearbeitung des Krankengutes und der Literatur zur medikamentösen und chirurgischen Therapie des partalen pHPT, Nachteil die begrenzte Fallzahl und vor allem der fehlende Hinweis auf die Anzahl der potenziell im 2. Trimenon chirurgisch behandelbaren Patientinnen. Es ergibt sich jedoch aufgrund der vorliegenden Ergebnisse und der Literaturanalyse, dass das Präeklampsierisiko als Hauptrisiko der medikamentösen Therapie gegenüber der chirurgischen Therapie erhöht ist und dies vor allem bei Kalziumwerten von $>3 \mathrm{mmol} / \mathrm{l}$. Angesichts des heute insgesamt gegenüber früher deutlich niedrigeren Operationsrisikos in der Schwangerschaft sprechen die Daten der Studie daher für ein operatives Vorgehen beim partalen pHPT im 2. Trimenon, insbesondere bei ansteigenden und $>3 \mathrm{mmol} / \mathrm{l}$ liegenden Serumkalziumwerten.

\section{Korrespondenzadresse}

Prof. Dr. med. Dr. h. c. mult. Henning Dralle Sektion Endokrine Chirurgie, Klinik für Allgemein-, Viszeral- und Transplantationschirurgie, Universitätsklinikum Essen Hufelandstr. 55, 45147 Essen, Deutschland henning.dralle@uk-essen.de

Interessenkonflikt. H. Dralle und T. Weber geben an, dass kein Interessenkonflikt besteht. 